Applied Physics Letters, 2007, v90, (14), art no. 142901

\title{
Asymmetry of domain forward switching and multilevel relaxation times of domain backswitching in antiferroelectric
}

\author{
$\mathrm{Pb}_{0.99} \mathrm{Nb}_{0.02}\left(\mathrm{Zr}_{0.84} \mathrm{Sn}_{0.12} \mathrm{Ti}_{0.04}\right)_{0.98} \mathrm{O}_{3}$ thin films
}

\author{
A.Q. Jiang, Y.Y. Lin , T.A. Tang \\ ASIC \& System State Key Lab, Department of Microelectronics, Fudan University, Shanghai \\ 200433, China \\ Q. Zhang* \\ Cranfield University, Department of Materials, Cranfield, Bedfordshire, MK43 OAL, UK
}

$\mathrm{Pb}_{0.99} \mathrm{Nb}_{0.02}\left(\mathrm{Zr}_{0.84} \mathrm{Sn}_{0.12} \mathrm{Ti}_{0.04}\right)_{0.98} \mathrm{O}_{3}$ thin films demonstrate a double hysteresis loop characteristic of antiferroelectric behavior with film thickness at above $150 \mathrm{~nm}$, but only one branch of the loop is present in films that are $90 \mathrm{~nm}$ thick. The relaxation time of domain backswitching from ferroelectric into antiferroelectric is multi staged in a range of $100 \mathrm{~ns}-100 \mathrm{~ms}$, but it is less than $100 \mathrm{~ns}$ in the films without $\mathrm{Sn}$ and $\mathrm{Nb}$ dopants. Electrical modeling of the films composed of elementary regions with inhomogeneities of dielectric constant, conductivity, and polarization embodies the essence of charge injection for the compensation of local backswitching field of domains.

Pacs Numbers: 77.80.-e, 77.22.Ej, 77.55.+f

Antiferroelectric materials have been studied extensively over the past several decades due to their applications in high-energy storage capacitors, high strain actuators/transducers, pyroelectric thermal sensing, electrocaloric cooling, and

\footnotetext{
* The author to whom the correspondence should be addressed, email: Q.Zhang@Cranfield.ac.uk.
} 
decoupling capacitors in multichip modules (MCMs). ${ }^{1-5}$ In an antiferroelectric (AF), an antiparallel ordered array of local dipoles can be switched into a ferroelectric (FE) at fields larger than a coercive field of $E_{\mathrm{AF}-\mathrm{FE}}$. The field-induced FE phase would revert back into its original state when the applied field is below a coercive field of $E_{\mathrm{FE}-\mathrm{AF}}$, releasing all of their polarization charges in a few nanoseconds, ${ }^{1}$ which facilitates the microelectronic devices operated at a very high speed. In most cases, $E_{\mathrm{AF}-\mathrm{FE}}$ is extremely high in thin films that require a high voltage-power supply for the device operation. With the development of present thin-film deposition technologies, the operating voltage can be greatly reduced when the film thickness is reduced. However, the vertical shrinkage of film thickness would result in the size effect: the films become a ferroelectric below a critical thickness. In general, the critical film thickness depends on the type of material as well as the fabrication technique, for example the critical thickness is around $400 \mathrm{~nm}$ for $\mathrm{PbZrO}_{3}$ films made by pulsed-laser deposition and $210 \mathrm{~nm}$ for $\mathrm{Pb}_{0.97} \mathrm{La}_{0.02}\left(\mathrm{Zr}_{0.85} \mathrm{Sn}_{0.13} \mathrm{Ti}_{0.02}\right) \mathrm{O}_{3}$ by sol-gel processing. ${ }^{6-7}$ The fundamental understanding of the size effect is necessary for the optimization of film fabrication, which is otherwise different from that on the long-range coupling of ferroelectricity to occur at a much smaller size. ${ }^{8}$

An alternative way to reduce $E_{\mathrm{AF}-\mathrm{FE}}$ is to alter the $\mathrm{PbZrO}_{3}$ composition in the phase diagram, such as the partial replacement of $\mathrm{Zr}$ by $\mathrm{Sn}$ in $\mathrm{Pb}_{0.97} \mathrm{La}_{0.02}\left(\mathrm{Zr}_{0.65} \mathrm{Sn}_{0.22} \mathrm{Ti}_{0.13}\right)_{3}$ ceramics. ${ }^{3}$ However, the field-induced FE phase is stable over the experimental period even after removal of the external field and exhibits a slow relaxation mechanism at room temperature. The slow relaxation 
kinetics is reminiscent of the domain backswitching in epitaxial ferroelectric thin films where the domains are preferentially orientated after the application of an electrical field. ${ }^{9-10}$ After the specimen is heated, the relaxation process into the antiferroelectric is accelerated, as evidenced from observations of transmission electron microscopy (TEM) micrographs. ${ }^{3}$ The mechanisms responsible for the slow relaxation are currently unclear due to the limited amount of data, especially in antiferroelectric thin films.

In this letter, the AF-FE phase switching was examined. A charge injection model is used to show the FE-to-AF relaxation behavior.

In this study, a sol-gel spin coating technique was used to deposit antiferroelectric $\mathrm{Pb}\left(\mathrm{Zr}_{0.95} \mathrm{Ti}_{0.05}\right) \mathrm{O}_{3}$ and $\mathrm{Pb}_{0.99} \mathrm{Nb}_{0.02}\left(\mathrm{Zr}_{0.84} \mathrm{Sn}_{0.12} \mathrm{Ti}_{0.04}\right)_{0.98} \mathrm{O}_{3}$ (PNZST) thin films onto $\mathrm{Pt} / \mathrm{Ti} / \mathrm{SiO}_{2} / \mathrm{Si}$ substrates. The details of the fabrication of $\mathrm{Pb}\left(\mathrm{Zr}_{0.95} \mathrm{Ti}_{0.05}\right) \mathrm{O}_{3}$ thin films have previously been described in Ref. 4. For the PNZST thin films, lead acetate trihydrate was dissolved in acetic acid and refluxed for $2 \mathrm{~h}$. Zirconium propoxide, titanium butoxide, tin acetate and niobium ethoxide were mixed and dissolved in acetic acid and 2-methoxyethanol in a separate flask. The two solutions were then mixed at room temperature and refluxed for $3 \mathrm{~h}$. De-ionized water and ethylene glycol were then added to control the viscosity and cracking of the films. The final concentration of the solution was $0.3 \mathrm{M}$. A layer was spin coated onto a substrate at $4000 \mathrm{rpm}$. The deposited layer was initially heated to $300{ }^{\circ} \mathrm{C}$ for $30 \mathrm{~s}$ and then annealed at $600{ }^{\circ} \mathrm{C}$ for $10 \mathrm{~min}$. The deposition procedure was repeated until the required film thickness was reached. Top electrodes of $\mathrm{Cr} / \mathrm{Au}$ were vacuum 
evaporated onto the films, and the electrodes were patterned into $60 \mu \mathrm{m} \times 60 \mu \mathrm{m}$ squares using photo-resist lift-off processing and wet chemical etching. All the films showed good crystallinity and perovskite structure, when examined using X-ray diffraction (XRD) patterns (data not shown). A Keithley 3402 waveform generator was used to supply various function-step voltage pulse sequences with a rise time of 5 ns, which allowed a variable pulse delay through two-channel configurations. The charge density of the domain forward/backward switching under/after the applied voltage $V_{\text {appl }}$, denoted as $P_{\mathrm{FSW}} / P_{\mathrm{NSW}}$, was analyzed through charge integration of time-dependent transient current across the film in series with a loading resistor of $R_{\mathrm{L}}$ $=50 \Omega$ monitored by an LeCroy WR 6200A oscilloscope. Each measurement was an average of 10 readings. $P$-E hysteresis loops were performed on a RT66A Radiant tester with a triangular wave form of $1 \mathrm{kHz}$.

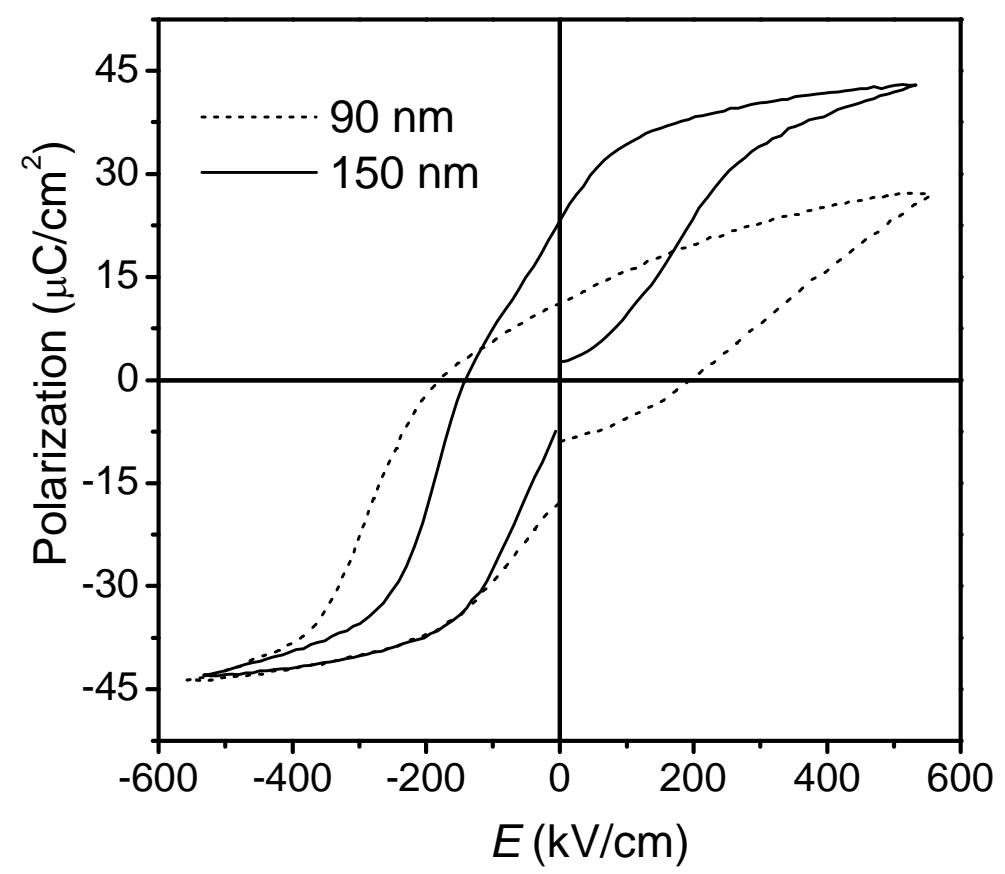

FIG. 1. P-E hysteresis loops for PNZST with different film thicknesses 
Fig.1 shows the $P-E$ hysteresis loops for PNZST thin films with different thicknesses. The coercive field $E_{\mathrm{AF}-\mathrm{FE}}$ for a typical $\mathrm{Pb}\left(\mathrm{Zr}_{0.95} \mathrm{Ti}_{0.05}\right) \mathrm{O}_{3}$ film ${ }^{4}$ is on the order of $500 \mathrm{kV} / \mathrm{cm}$, which is reduced to $\sim 200 \mathrm{kV} / \mathrm{cm}$ for a $150 \mathrm{~nm}$-thick PNZST film due to ionic replacement here. The loop appears similar to that from other ferroelectric material in agreement with previous reports. ${ }^{3,11}$ As the film thickness is reduced to $90 \mathrm{~nm}$, the loop at negative fields on the top electrode remains, but half of the loop at positive fields is lost, as shown in Fig. 1. This indicates that the "ferroelectric" domains driven by $E_{\mathrm{FE}-\mathrm{AF}}$ have a preferred orientation pointing to the top electrode.

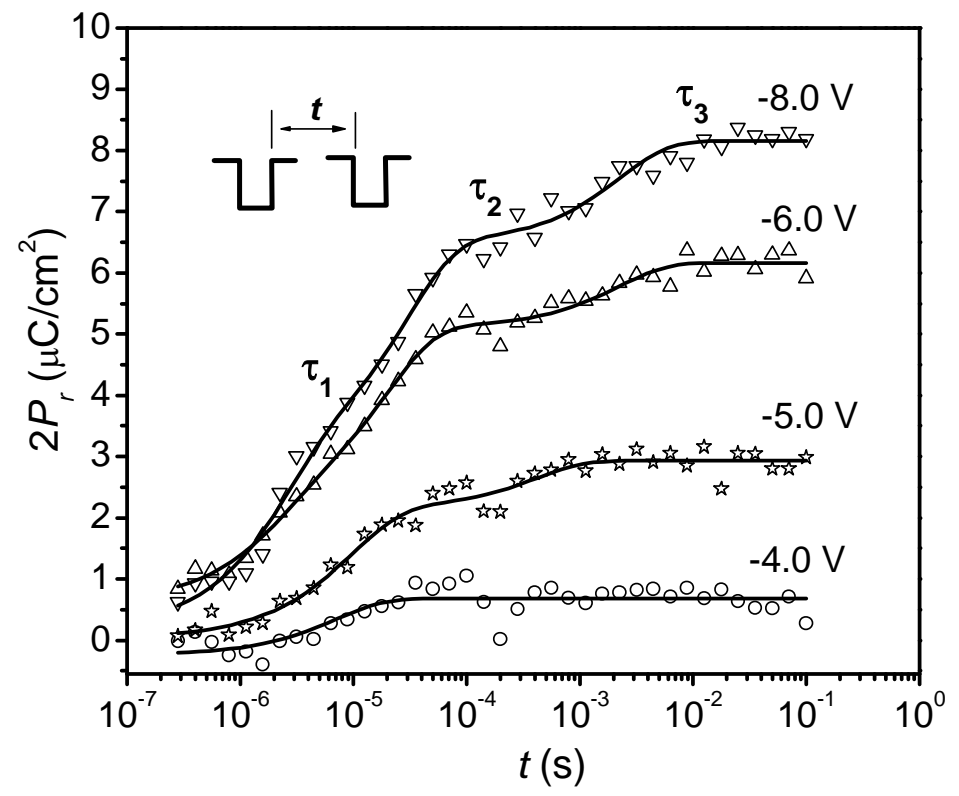

FIG. 2. Time dependence of remanent polarization under a negativenegative pulse sequence_inset_separated with relaxation time t under different Vappl for PNZST. The pulse width is $100 \mathrm{~ns}$. The solid lines are the best fitting of the data according to Eq. _5_. 


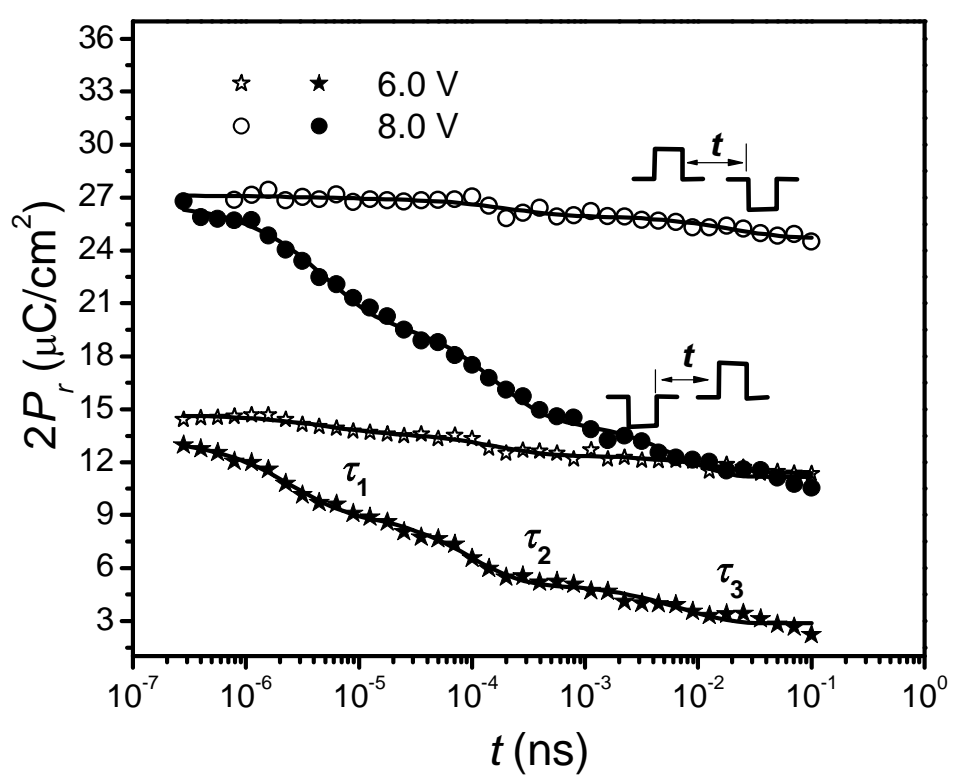

FIG. 3. Time dependence of remanent polarization under a positive-negative or negative-positive pulse sequence _opened or closed symbols_separated with relaxation time t under different_Vappl_for PNZST. The pulse width is $100 \mathrm{~ns}$. The solid lines are the best fitting of the data according to Eq. _5_.

Figure 2 shows the time dependence of the remanent polarization $2 P_{r}(t)=\left|P_{\mathrm{FSW}}(t)\right|-$ $\left|P_{\mathrm{BSw}}(t)\right|$ after a negative-negative pulse sequence with different $V_{\text {appl. }} .2 P_{r}(t)$ values increases with the increase of either relaxation time $t$ or $\left|V_{\text {app }}\right|$. The time evolution of $2 P_{r}$ at each $V_{a p p l}$ is characterized by multiple steps, implying that there are several relaxation mechanisms. As the polarity of the pulses in the sequence are firstly positive and then negative, $2 P_{r}$ is nearly constant with time, as shown in Fig. 3, but it decays with time to show the same relaxations as those in Fig. 2 under a reversed pulse sequence. This supports the preferred domain orientation after $V_{\text {appl }}$.

When the above pulse sequences are applied on a $350 \mathrm{~nm}$-thick $\mathrm{Pb}\left(\mathrm{Zr}_{0.95} \mathrm{Ti}_{0.05}\right) \mathrm{O}_{3}$ thin film, both $\left|P_{\mathrm{FSW}}\right|$ and $\left|P_{\mathrm{BSW}}\right|$ are nearly constant in the time range of $100 \mathrm{~ns}-100$ ms with almost zero remanent polarization. This suggests that in physical nature, 
phase switching from $\mathrm{FE}$ to $\mathrm{AF}$ can be fast, and that the slow restoration of an antiferroelectric phase in the films is attributed to additional factors, such as the size effect and film heterogeneity arising from regional defect accumulation, strain-field distribution, phase segregation, and interfacial passive layers. ${ }^{6-7,12-13}$ The generic size effect in antiferroelectric thin films can cause: (1) the gradual increase of the remanent polarization with the decrease of the film thickness, ${ }^{7}$ and (2) the abrupt transformation into a ferroelectric at below a critical film thickness. ${ }^{6,14}$ In our work asymmetric phase switching under positive and negative fields is exceptional and still under investigation. It is suggested that the preferable defect accumulation at one side of the film eliminates the reverse domain nucleation and thus inhibits AF-to-FE phase switching at positive fields. Nevertheless, the size effect can not explain the slow relaxation of polarization reversal, since the same phenomenon can occur in bulk ceramics. $^{3}$

As a reasonable assumption, the regional heterogeneous nature of the film can affect local electrical parameters of polarization $\left(P_{i}\right)$, dielectric permittivity $\left(\varepsilon_{i}\right)$, and conductivity $\left(\sigma_{i}\right)$ in an elementary region $(i)$ with the thickness $\left(d_{i}\right)$ that causes charge accumulation at the interface under $V_{\text {appl }}$. From dielectric continuum equations, we can state

$$
\begin{aligned}
& D_{i}=\varepsilon_{0} E_{i}+P_{i}=\varepsilon_{0} \varepsilon_{i} E_{i}+P_{s}^{(i)}, \\
& V_{a p p l}=\sum_{i} d_{i} E_{i},
\end{aligned}
$$




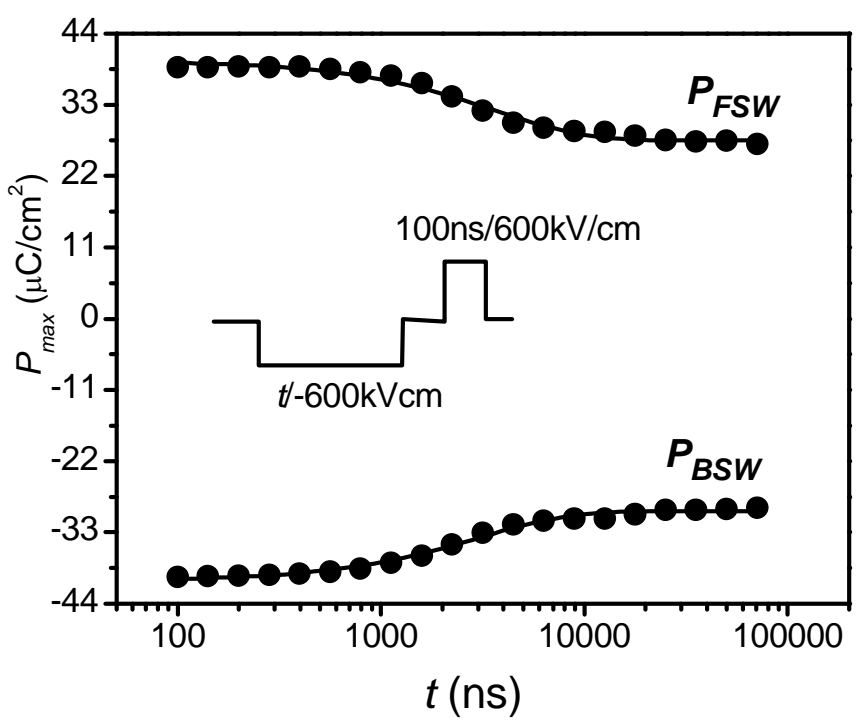

FIG. 4. Storing and releasing charge density dependences of $V$ appl stressing time for domain switching from AF-to-FE and FE-to-AF states for a

350-nm-thick Pb_Zro.95Tio.05_O3 film. The solid lines are the data fits according to Eq._4_.

and $J_{i}=\sigma_{i} E_{i}+\frac{\partial D_{i}}{\partial t}$

where $P_{s}^{(i)}, D_{i}, E_{i}$ and $J_{i}$ are the saturation polarization, the dielectric displacement, the electric field, and the current density in the region $i$, respectively, and $\varepsilon_{0}$ is the vacuum permittivity. In a film consisting of two parallel-plate regions $(i=2)$ connected in series, we can calculate the interfacial charge density using

$q_{1}(t)=\left[\frac{\varepsilon_{0} \varepsilon_{1} \sigma_{2} d_{2}+\varepsilon_{0} \varepsilon_{2} \sigma_{2} d_{1}}{\sigma_{2} d_{1} d_{2}+\sigma_{1} d_{2}^{2}} V_{a p p l}-\frac{\varepsilon_{0} \varepsilon_{2}}{d_{2}} V_{a p p l}+\left(P_{s}^{(1)}-P_{s}^{(2)}\right)\right]\left[1-\exp \left(-\frac{t}{t_{1}}\right)\right]$

without considering $\varepsilon_{i}$ and $\sigma_{i}$ dependences of the local field $E_{i}$, where $t_{1}=\frac{\varepsilon_{0} \varepsilon_{1} d_{2}+\varepsilon_{0} \varepsilon_{2} d_{1}}{\sigma_{1} d_{2}+\sigma_{2} d_{1}}$. The injected charges under $V_{\text {appl }}$ can not only compensate for the local driving field $E_{\mathrm{FE}-\mathrm{AF}}$ in prohibiting domain backswitching but also cause pinning of domain walls. ${ }^{15}$ After the removal of $V_{\text {appl }}$, the injected charges can be smoothed out of the film and then $-E_{\mathrm{FE}-\mathrm{AF}}$ for domain-wall motion is restored. The process is 
characterized by a relaxation time $\left(t_{1}\right)$ that is dependent on the regional dielectric constant, conductivity and thickness. Figure 4 shows $P_{\mathrm{FSW}}$ and $P_{\mathrm{BSW}}$ profiles for $\mathrm{Pb}\left(\mathrm{Zr}_{0.95} \mathrm{Ti}_{0.05}\right) \mathrm{O}_{3}$ thin films after negative field stressing for different times. Their absolute values both decrease with a prolonged time until leveling off at a characteristic time, $t_{1}$. However, their polarizations are completely restored after a programmed 1 second relaxation period. The solid lines of best fits of the data in Fig. 4 yield $t_{1}=3.18 \mu \mathrm{s}$, where polarization reduction is assumed to be proportional to the injected charge density.

It is expected that the heterogeneous regions would be enhanced in doped PNZST thin films, due to the increase of defect centers by ionic replacements, and the relaxation time would change from region to region with regard to the electrical parametric variation. The total injected charge density under $V_{\text {appl }}$ would be

$$
q(t)=\sum_{i} q_{i}(t)
$$

The line of best fit suggests that either polarization increase in Fig. 2 or reduction in Fig. 3 is proportional to the total charges. Three discrete relaxation times in the spectra are clearly identified to be $t_{1}=2.31 \mu \mathrm{s}, t_{2}=29.7 \mu \mathrm{s}$ and $t_{3}=2.23 \mathrm{~ms}$ at $\left|V_{\text {appl }}\right|=$ $8.0 \mathrm{~V}$ in our experimental range, which all slightly decrease when the $\left|V_{\text {appl }}\right|$ is reduced.This can be understood from the nature of the field-dependent $\varepsilon_{i}$ and $\sigma_{i}$ in a nonlinear dielectric material. With the charges smoothed out of the film after $V_{\text {appl }}$, the local driving force $E_{\mathrm{FE}-\mathrm{AF}}$ is restored for the reverse domain nucleation with an area fraction of $p_{i}(t)=1-\exp \left(-t / \tau_{i}\left(E_{i}\right)\right) .{ }^{16}$ If $\left\langle\tau_{i}\left(E_{i}\right)\right\rangle>t_{i}$, the polarization relaxation can be more accurately described by a pre-exponential formula, such as in epitaxial 
ferroelectric $\mathrm{Pb}(\mathrm{Zr}, \mathrm{Ti}) \mathrm{O}_{3}$ thin films, ${ }^{9-10}$ due to the consideration of nucleation-limited domain switching in a low dimensional system with an effective Hausdorff dimensionality of $d=2.5 .^{17-18}$

In summary, the coercive field in antiferroelectric $\mathrm{Pb}\left(\mathrm{Zr}_{0.95} \mathrm{Ti}_{0.05}\right) \mathrm{O}_{3}$ thin films can be reduced by doping with $\mathrm{Nb}$ and $\mathrm{Sn}$ ions. However, a double $P-E$ hysteresis loop would lose its positive branch as the film thickness is below $90 \mathrm{~nm}$ due to the size effect, and the remaining part exhibits a preferred "ferroelectric" domain orientation driven by $E_{\mathrm{FE}-\mathrm{AF}}$. The relaxation times of domain backswitching are multiple in the range of $100 \mathrm{~ns}$ to $100 \mathrm{~ms}$, reminiscent of its slow behavior in the bulk, ${ }^{3}$ but fast into the order of a few nanoseconds in the film without dopants. It is believed that the local-field rejuvenation after injected charge compensation for $E_{F E-A F}$ is essential for multiple polarization relaxations in the film consisting of elementary regions with different electrical parameters.

Acknowledgement This work is supported by Climbing Mountain Plan of Shanghai in Project No. 06JC14006 and NCETFDU. Dr Zhang would like to thank the EPSRC financial support (grant GR/S 45027/01). 


\section{References}

1. B. Xu, P. Moses, N.G. Pai, and L.E. Cross, Appl. Phys. Lett. 72, 593 (1998).

2. D. Berlincourt, H. Jaffe, H.H.A. Krueger, and B. Jaffe, Appl. Phys. Lett. 3, 90 (1963).

3. W. Chan, Z. Xu, J. Zhai, and H. Chen, Appl. Phys. Lett. 87, 192904, (2005).

4. A. Mischenko, Q. Zhang, J. F. Scott, R. W. Whatmore, and N. D. Mathur, Science 311, 1270 (2006).

5. C.J. Gaskey, K.R. Udayakumar, H.D. Chen, and L.E. Cross, J. Mater. Res. 10, 2764 (1995).

6. P. Ayyub, S. Chattopadhyay, R. Pinto, and M.S. Multani, Phys. Rev. B 57, R5559 (1998).

7. B. Xu, Y. Ye, Q. Wang, and L.E. Cross, J. Appl. Phys. 85, 3753 (1999).

8. J. Junquera and Ph. Ghosez, Nature 422, 506 (2003).

9. C.S. Ganpule, V. Nagarajan, S.B. Ogale, A.L. Roytburd, E.D. Williams, and R. Ramesh, Appl. Phys. Lett. 77, 3275 (2000).

10. C.S. Ganpule, A.L. Roytburd, V. Nagarajan, B.K. Hill, S.B. Ogale, E.D. Williams, R. Ramesh, and J.F. Scott, Phys. Rev. B 65, 14101 (2001).

11. Z. Jiwei, M. H. Cheung, Z. Kui Xu, X. Li, H. Chen, E. V. Colla, and T. B. Wu, Appl. Phys. Lett., 81, 3621 (2002).

12. J.M. Benedetto, R.A. Moore, and F.B. McLean, J. Appl. Phys. 75, 460 (1994).

13. A.Q. Jiang, Y.Y. Lin, and T.A. Tang, J. Appl. Phys. 2007 (ID: 131703JAP, in press). 
14. I. Kanno, S. Hayashi, M. Kitagawa, R. Takayama, and T. Hirao, Appl. Phys. Lett. 66, 145 (1995).

15. A.Q. Jiang, Y.Y. Lin, and T.A. Tang, Appl. Phys. Lett. 89, 32906 (2006).

16. A.K. Tagantsev, I. Stolichnov, and N. Setter, Phys. Rev. B 66, 214109 (2002).

17. P. Paruch, T. Giamarchi, and J.-M. Triscone, Phys. Rev. Lett. 94, 197601 (2005).

18. J.F. Scott, J. Phys.: Condens. Mat. 18, 7123 (2006)) 


\section{Figure Captions:}

1. $P-E$ hysteresis loops for PNZST with different film thicknesses.

2. Time dependence of remanent polarization under a negative-negative pulse sequence (inset) separated with relaxation time $t$ under different $V_{\text {appl }}$ for PNZST. The pulse width is $100 \mathrm{~ns}$. The solid lines are the best fitting of the data according to Eq. (5).

3. Time dependence of remanent polarization under a positive-negative or negative-positive pulse sequence (opened or closed symbols) separated with relaxation time $t$ under different $\left|V_{\text {appl }}\right|$ for PNZST. The pulse width is 100 ns. The solid lines are the best fitting of the data according to Eq. (5).

4. The storing and releasing charge density dependences of $V_{\text {appl }}$ stressing time for domain switching from AF-to-FE and FE-to-AF states for a $350 \mathrm{~nm}$ - thick $\mathrm{Pb}\left(\mathrm{Zr}_{0.95} \mathrm{Ti}_{0.05}\right) \mathrm{O}_{3}$ film. The solid lines are the data fitting according to Eq. (4). 